Article

\title{
(A)morally Demanding Game? An Exploration of Moral Decision-Making in a Purpose-Made Video Game
}

\author{
Sarah E. Hodge *, Jacqui Taylor and John McAlaney \\ Department of Psychology, Bournemouth University, Poole, BH12 5BB, UK; E-Mails: shodge@bournemouth.ac.uk (S.E.H.), \\ jtaylor@bournemouth.ac.uk (J.T.),jmcalaney@bournemouth.ac.uk (J.M.) \\ * Corresponding author
}

Submitted: 27 June 2019 | Accepted: 18 October 2019 | Published: 20 December 2019

\begin{abstract}
A purpose-made video game was used to measure response time and moral alignment of in-game moral decisions, which were made by 115 undergraduate students. Overall, moral decisions took between 4-6 seconds and were mostly pro-social. Previous gameplay, in-game, and post-game experiences predicted in-game moral alignment. Real-life moral salience was not related to in-game decision-making. The implications of these results are discussed in the context of the demands of video games and in-game moral decision-making models.
\end{abstract}

\section{Keywords}

decision-making; digital games; moral foundations theory; morality; purpose-made games; video games

\section{Issue}

This article is part of the issue "Video Games as Demanding Technologies" edited by Nicholas David Bowman (Texas Tech University, USA).

(C) 2019 by the authors; licensee Cogitatio (Lisbon, Portugal). This article is licensed under a Creative Commons Attribution 4.0 International License (CC BY).

\section{Introduction}

One of the distinct features of video games is their interactivity (Bowman, 2018; Tamborini \& Bowman, 2010). Recent research has suggested that interactivity is a process, involving the interaction between player and game (Bowman, 2018; Rutledge, 2013; Stromer-Galley, 2004). Moral decisions in video games demonstrate this process of interactivity between player and game; many in-game moral decisions often require players to choose from options which effect in-game outcomes. Furthermore, it has been suggested that this interactivity leads to different requirements, termed as the 'demands' of video games, which are categorized into types of efforts: social, emotional, cognitive, and two types of behavioral; exertion and controls (Bowman, 2016, 2018; Bowman, Wasserman, \& Banks, 2018). Hence, the demands of video games connect well to morality and has implications for in-game behavior, as real-life morality can be examined through components such as moral judgment and action (Haidt \& Joseph, 2008; Narvaez \& Rest,
1995). Moral action could be related to the behavioral demands, especially the control demand (i.e., physically pushing a button to make a decision). Eden, Ewoldsen, Lee, and Beyea (2018) highlighted how physical demands are more than just pressing a button and can be behavioral affordances; which is how the individual perceives and behaves within games (i.e., the action[s] that can be taken, and how options are presented and sequenced). Previous methodological issues in moral decision-making research have been highlighted, for example, the accuracy of self-reported morality due to the gaps between moral judgment and action (American Psychological Association, 2015; Haviv \& Leman, 2002). The benefits of exploring in-game decisions are that behavioral demands/affordances can be directly measured (Eden et al., 2018; Hodge et al., 2018).

Much of the previous research has used commercial games which can restrict aspects of the moral decisionmaking process and create biases, such as reward systems (Hodge et al., 2018). Some previous research attempted to addressed this, with a modified version of 
a commercial game (Joeckel, Bowman, \& Dogruel, 2012, 2013). Modifying commercial games allows for these preexisting games to be altered and adapted for research purposes (Elson \& Quandt, 2016; Hartmann, Toz, \& Brandon, 2010; Mohseni, Liebold, \& Pietschmann, 2015). However, there are still some limitations to modifying commercial games such as restrictions and biases. To further address some of these limitations, purpose-made games and Virtual Environments (VE) can be developed for research (Hartmann et al., 2010; Hodge et al., 2018; Huskey, Craighead, Miller, \& Weber, 2018). A purposemade game is a game created to a specification for research, in this instance a theory driven bespoke game was developed in a game engine to measure and record in-game moral decisions (Hodge et al., 2018). Game engines are well-suited for research as they provide complete control over the environment and the design of the game/VE (Lewis, \& Jacobson, 2002). The present study aimed to explore the results of the data collected from the purpose-made game and to understand the in-game moral decision-making process.

Tamborini (2011, 2012) proposed the Model of Intuitive Morality and Exemplars (MIME) to explain the decision-making process with media, including video games. The MIME adopts the six Moral Foundations Theory (MFT) domains of: Care/Harm (C/H); Fairness/Cheating (F/C); Authority/Subversion (A/S); Sanctity/Degradation (S/D); In-group Loyalty/Betrayal (L/B); and Liberty/Oppression (L/O). These MFT domains are composed of virtues, triggers, emotions, and cognition; with moral decisions consisting of both intuitive and rational processes (Haidt, 2012; Haidt \& Joseph, 2008). The MIME suggests moral judgment happens through media activating and influencing the moral domains salience (importance and hierarchy). Then intuitive/automatic processing of the media takes place, unless in conflict (e.g., moral violation or competing domains), where reflective and deliberation processes are activated to try and resolve the conflict, but this does not necessarily lead to a change in decision (Tamborini, 2011, 2012). This model draws on the dual-process theories; system 1, quick experiential, and system 2, slower rational (Hartmann, 2011; Kahneman, 2011).

\subsection{Rationale}

Tamborini, Bowman, et al. (2016, p. 13) highlight that research needs to develop a "dynamic understanding of in-game decisions." Hence, through synthesizing the research on MFT, MIME, with the demands of video games, creates much value for the research, helping to understand the context and the theories that underpin in-game moral decision-making. Thus, the contribution of this research aims to explore aspects of the in-game decision-making process within a purpose-made game.

Time is used frequently in gameplay, such as requiring the player to respond with speed, as seen in fighting/beat 'em up games, and as a design feature, such as timers and countdowns (Palmer, 2015; Swink, 2009). Sensory input to muscle response takes around 240 milliseconds (Swink, 2009). Moral intuitive decisions have been suggested to take around 500-1500 milliseconds (e.g., Tamborini, Lewis, et al., 2016), but there has been little research which has explored Response Time (RT) for in-game moral decisions. Furthermore, considering the dual-process theories (e.g., Hartmann, 2011), RT could add valuable insight into the in-game decision-making process. Therefore, questions still remain around the players response times to in-game moral decisions:

\section{RQ1: How long do in-game moral decisions take?}

Moral positioning or alignment can represent in-game behavior-how good, evil, or neutral the players have chosen to be (Triberti, Villani, \& Riva, 2015). Much of the previous research has focused on post-game effects including post-game moral behavior (American Psychological Association, 2015; Gollwitzer \& Melzer, 2012; Saleem, Anderson, \& Barlett, 2015). Ellithorpe, Cruz, Velez, Ewoldsen, and Bogert (2015) highlighted the reciprocal relationship between in-game moral decisionmaking and post-game behavior, through the process of moral licensing, that is, the rationalisation of allowing immoral behavior due to previous good behavior. The results suggested that if participants perceived the gameplay to be moral this predicted more anti-social behavior post-game (Ellithorpe et al., 2015). Other research has found previous gameplay experiences to be related to in-game behavior/experience such as players becoming morally disengaged in gameplay (Hartmann, Krakowiak, \& Tsay-Vogel, 2014; Hartmann \& Vorderer, 2010; Lange, 2014). Joeckel et al. (2013) found enjoyment was related to presence for both US and German participants, and gaming experience for US participants. However, it would have been interesting to know if presence and previous gaming experience related to in-game moral decisions. Previous research has also shown some overlap between in-game and real-life morality wherein choices were suggested to mirror real-life morality and a "strong moral presence" was found in video games (Weaver \& Lewis, 2012, p. 613). Hence, more research is needed to understand what factors predict in-game moral decision-making:

RQ2: Which factors of real-life MFT domains, previous gameplay, in-game and post-game experiences, will predict in-game moral alignment?

Real-life moral salience has been suggested to relate to in-game choices, particularly, the $\mathrm{C} / \mathrm{H}$ domain which has been suggested to be intuitive (Joeckel et al., 2012, 2013; Tamborini, Bowman, et al., 2016). Joeckel et al. $(2012,2013)$ also suggested that non-salient domains were related to amoral decision-making (without morality which can have connotations of immorality). Consequently, more research is needed to understand 
the connection between real-life moral salience and ingame moral choices:

RQ3: How will real-life moral salience relate to ingame moral choice and RT?

\section{Method}

\subsection{Design}

A mixed design quasi-experiment was used. The two dependent variables were the moral decisions from each of the 6 in-game MFT scenarios (see Appendix A):

1) The choices made (upholding/pro-social or violating/anti-social, for each in-game MFT scenarios). In-game moral alignment was calculated by subtracting violating choice from the upholding choice. A negative score equals more violating choices made and a positive score equals more upholding choices made.

2) RT was measured in milliseconds for each of the 6 MFT in-game decisions.

The predictor variables were divided into three groups real-life morality, previous gameplay, in-game and postgame questions (Appendix B).

\subsection{Participants}

115 undergraduate students took part and were recruited through opportunity and volunteer sampling ( $M$ age =19.96; $S D=2.84)$. One participant withdrew during gameplay and thirteen were excluded for the following reasons: Moral Foundations Questionnaire (MFQ) exclusion criteria (two questions to measure inattention); leaving the room during the gameplay; and technical problems with the purpose-made game. This reduced the total to 101 participants. The majority reported a white background (78\%), with the gender of the sample being $45 \%$ male. Ethical approval was obtained from the University's Research Ethics team, carried out within accordance of the University's Research Ethics Code of Practice.

\subsection{Materials}

The materials listed below in order of completion included the developed purpose-made game (Hodge et al., 2018) and the measures used in the questionnaire. The questionnaire was administered through an online survey tool, SurveyMonkey.

\subsubsection{Pre-Game Questions}

The MFQ32 (Graham, Haidt, \& Nosek, 2008) created scores for each of the MFT domains and were calcu- lated from a 6-point scale: $\mathrm{C} / \mathrm{H} \alpha=.69 ; \mathrm{F} / \mathrm{C} \alpha=.65$; L/B $\alpha=.71 ; \mathrm{A} / \mathrm{S} \alpha=.74 ; \mathrm{S} / \mathrm{D} \alpha=.84$ (Graham et al., 2011); and L/O $\alpha=.62$ (Hodge, 2018; Appendix C). These reliability alphas are only satisfactory/acceptable (Taber, 2018). It is acknowledged that the reliability scores have an effect on analyzing the data; therefore, calculating moral salience can support addressing this, as well as understanding the hierarchical structure of the MFT domains. Plus, moral salience has been suggested to underpin moral decision-making and was calculated by isolating both the highest and lowest scoring real-life MFQ domain(s) (Joeckel et al., 2012, 2013). Hence, categorical dummy variables represented if the MFQ domain was salient or non-salient (Yes/No) which was identified by manually isolating participant's highest and lowest scoring domains.

Participants were asked questions about their previous gameplay (Appendix B), which included the following questions: plays video games (Yes/No); if they would describe themselves as a gamer (Yes/No); length of time playing ( $0-52.50$ hours); years playing games (0-26 years); number of genres played (0-19); experience and ability (1 = Beginner/a little, to 7 = Expert/much).

\subsubsection{Purpose-Made Game}

Using the Unreal engine, the game was a 3D photorealistic first-person, role-playing game played on a PC. Each of the MFT foundations were represented by previously tested scenarios, located in separate rooms within the level (see Figure 1; Hodge et al., 2018). Once participants had located the Non-Player Character (NPC), the relevant MFT scenario was presented, then participants selected one of the two choices presented, either uphold/pro-social choice or violated/anti-social choice. For more information on the design of the purposemade game, see Appendix A, Hodge et al. (2018), and Hodge (2018).

\subsubsection{Post-Game Questions}

Participants were asked to select a preferred moral alignment in their previous gameplay (Good/Bad/Neutral) that were coded (Yes/No). Although a previous gameplay question, to avoid bias, participants were asked this after playing the purpose-made game.

Avatar attachment and empathy questions were taken from the Temple Presence Inventory and rated on the following scale: $1=$ Not at all, to $7=$ Very much ( $\alpha .=84$; Lombard, Ditton, \& Weinstein, 2007; see Appendix B).

An adapted Game Engagement Questionnaire (GEQ), which consists of 19 questions $(\alpha=.85)$ and rated on the following scale: Yes $=2$; Maybe $=1$; and $\mathrm{No}=0$ (Brockmyer, et al., 2009), was used to measure the level of engagement in the purpose-made game.

The Positive Affect $(\alpha=.88)$ and Negative Affect $(\alpha=.85)$ Schedule (PANAS-X) each contained 10 items, 


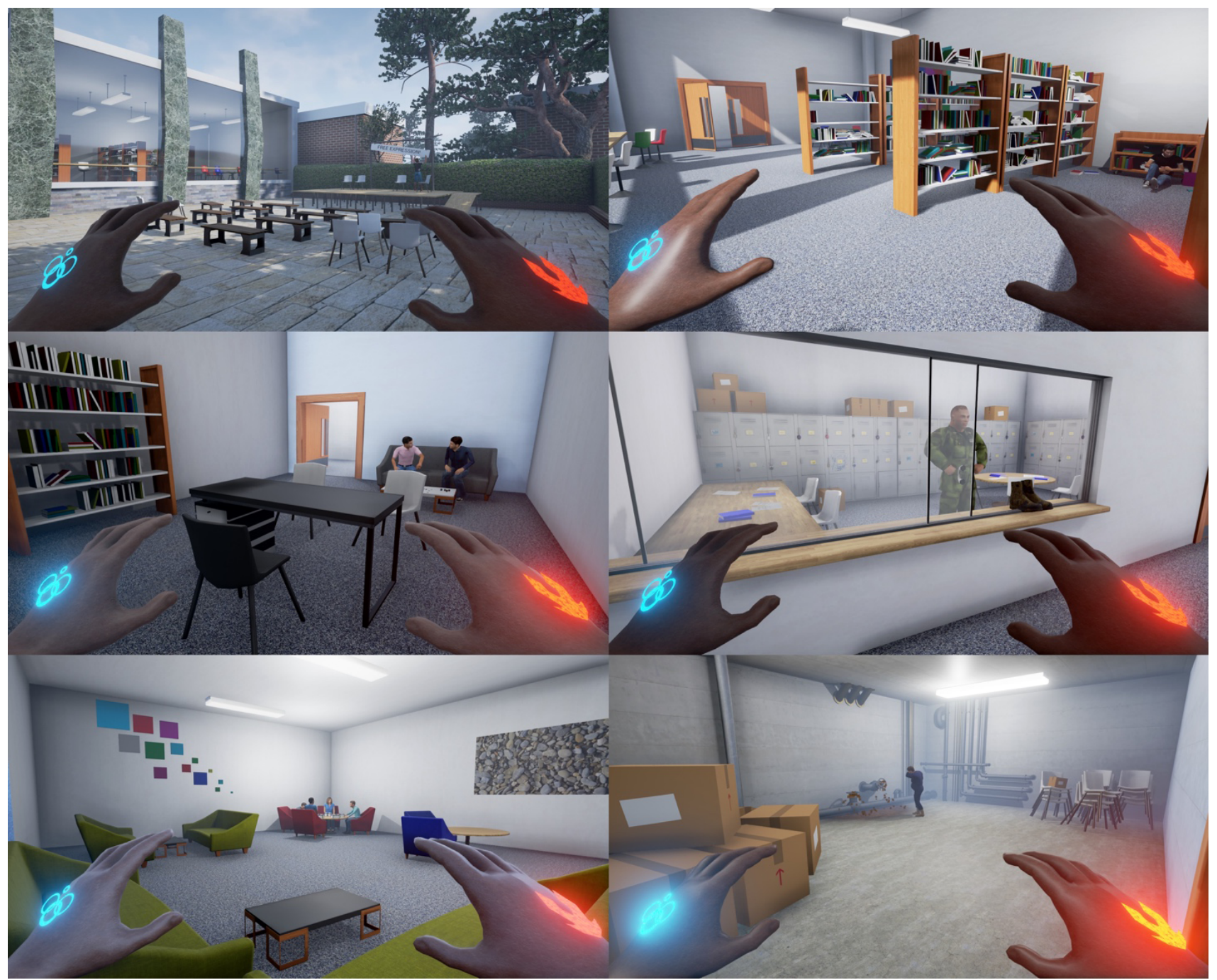

Figure 1. The six in-game MFT scenarios. Note: Top left clockwise-L/O, C/H, A/S, S/D, L/B, and F/C.

with the sub-measure Guilt score obtained from 6 items within the Negative Affect scale. The scale range included: 1 = Very slightly, to 5 = Extremely (Watson \& Clark, 1999).

The Tangram help/hurt task was used to measure post-game pro-social and anti-social behavior. Participants were led to believe they were assigning and completing tangrams puzzles with a (fictitious) participant and if the other participant could complete 11 tangrams in 10 minutes, they won a prize. Participants selected how easy or difficult they made the task. A helping score was calculated from the number of easy puzzles greater than one, with the same process used for calculating the hurting score for hard puzzles. A help/hurt score (Tangram alignment) was calculated by subtracting the helping from the hurting scores (Saleem et al., 2015).

\subsection{Procedure}

Minor deception was used as participants were told the research involved decision-making, with the moral aspects of the research explained in the debrief. All data was collected in the laboratory in one session. Once the consent form had been signed the first questionnaire commenced (see Section 2.3.1). Then the purpose-made game was played in the lab. Once the game had finished participants completed the second questionnaire (see Section 2.3.3). Finally, the Tangram task was completed, followed by a debrief.

\section{Results}

To explore how moral decisions were made in a purposemade game, Table 1 shows that in-game moral alignment was more pro-social and upholding (5 out of 6 domains) with RT on average taking at least 4 seconds. L/B then $\mathrm{F} / \mathrm{C}$ had the longest RTs, which could be due to the layout of the VE as these two scenarios were closest to the beginning of the level.

The RT data had a positive skew, therefore a reciprocal transformation was applied to reduce the effect of slow responses while keeping power in the data: inverting the data to the speed of the decision per millisecond (Whelan, 2008). In-game moral alignment was 
Table 1. Summary of descriptive statistics of the in-game moral decisions.

\begin{tabular}{|c|c|c|c|c|c|c|}
\hline In-game decisions & $M$ & $S D$ & $t$ & $d f$ & $p$ & $d$ \\
\hline \multicolumn{7}{|l|}{ In game choice } \\
\hline Upheld MFT (Pro-social) & 5.19 & 0.81 & & & & \\
\hline Violated MFT (Anti-social) & 0.81 & 0.94 & & & & \\
\hline In-game moral alignment & 4.38 & 1.87 & & & & \\
\hline \multicolumn{7}{|c|}{ RT (Seconds) by in-game MFT domain } \\
\hline L/B & 6.61 & 3.13 & -2.15 & 99 & .034 & -.43 \\
\hline $\mathrm{F} / \mathrm{C}$ & 5.60 & 2.50 & 1.41 & 99 & .161 & .28 \\
\hline $\mathrm{C} / \mathrm{H}$ & 4.09 & 2.65 & -3.22 & 6.28 & .017 & -2.57 \\
\hline$S / D$ & 4.01 & 1.78 & -2.56 & 99 & .012 & -.52 \\
\hline L/O & 4.24 & 2.18 & -3.60 & 99 & .719 & -.72 \\
\hline$A / S$ & 4.63 & 2.86 & 1.49 & 99 & .140 & .30 \\
\hline Total decision time & 29.33 & 10.17 & & & & \\
\hline Total time in level & 239.30 & 112.39 & & & & \\
\hline
\end{tabular}

significantly correlated with total decision time $r=.25$ $(p=.013)$, suggesting the quicker the decisions, the more decisions which were upheld/pro-social. Independent t-tests on the speed of decision and choice made suggested that upholding/pro-social choices were significantly faster than violating/anti-social choices for the domains of: $\mathrm{C} / \mathrm{H}$ with a large effect size; $\mathrm{S} / \mathrm{D}$ with a medium effect size; and L/B with a small effect size.

\subsection{RQ1: How Long Do In-Game Moral Decisions Take?}

A repeated measures ANOVA was conducted on speed of decision with each of the in-game MFT domains. Greenhouse-Geisser values are reported as Mauchly's test of sphericity was violated $\chi^{2}(14)=50.90(p<.001)$. Speed of decisions was significantly related to MFT domain $F(4.15,414.68)=21.87(p<.001) \eta^{2}=.18$. Post Hoc Bonferroni test suggests that the domain of in-game L/B and $\mathrm{F} / \mathrm{C}$ were significantly different $(p=.015)$ and slower than the other domains.

\subsection{RQ2: Which Factors of Real-Life MFT Domains, Previous Gameplay, In-Game, and Post-Game Experiences, Will Predict In-Game Moral Alignment?}

To explore what factors predicted moral alignment three multiple linear regressions were carried out. Table 2 shows that participants on average have been playing video games for around 7 years, with 6 types of genres and 6 hours of gameplay a week. Over two thirds of participants reported playing video games and a third identified as a gamer. Most of the participants wanted to help in the Tangram task. Avatar attachment and empathy were at the midpoint of the scale. Participants reported more negative affect than positive affect, and feelings of guilt were low.

To explore what factors predicted moral alignment, Table 3 shows the only significant predictor for the first regression was $\mathrm{C} / \mathrm{H}$, the model was not significant and only explained around $5 \%$ of the variance. The results of the second regression suggested the significant predictors were previous evil alignment and number of genres played had negative relationships with moral alignment whereas ability had a positive relationship. The third regression suggested empathy was a significant predictor with a positive relationship with in-game moral alignment whereas engagement had a significant negative relationship.

\subsection{RQ3: How Will Real-Life Moral Salience Relate to In-Game Moral Choice and RT?}

To explore the role of real-life moral salience and in-game decision-making, Table 4 shows the results of the participant's real-life moral salience listed in rank order. $\mathrm{C} / \mathrm{H}$ was the most salient, S/D was the lowest, and A/S received the most violations. Chi-squared analysis on reallife salience (low or high) and in-game choice (violate or upheld) for each MFT domain suggested none of the reallife MFT domains were significantly associated with ingame MFT scenarios, with Cramer's $V$ ranging from weak (S/D) to strong $(C / H)$. Independent t-tests on speed of decision and real-life salience (Yes/No), suggested only participants with a non-salient L/B foundation were significantly slower than those who did not have L/B as a non-salient foundation with a small effect size.

\section{Discussion}

The results suggested that in-game moral decisions took between 4-6 seconds, were mostly pro-social (in-game MFT domains upheld), with violations taking longer. The following predictor variables were suggested to have a significant relationship with in-game moral decisions: MFQ C/H score, evil alignment, ability, number of genres played, empathy, and engagement. Previous gameplay, in-game and post-game regression models did predict ingame moral decisions but not real-life morality. The MFT domains that were upheld and salient were similar to previous research (Joeckel et al., 2012, 2013; Tamborini, 
Table 2. Descriptive statistics of continuous and categorical predictor variables.

\begin{tabular}{lcc}
\hline Variables & M & SD \\
\hline Years playing & 7.63 & 5.96 \\
Number of genres played & 6.04 & 5.00 \\
Length of time & 6.61 & 8.22 \\
Gaming ability & 3.40 & 1.79 \\
Gaming experience & 3.59 & 2.01 \\
Tangram help score & 4.74 & 3.03 \\
Tangram harm score & 1.32 & 1.82 \\
Tangram alignment score & 3.43 & 4.56 \\
Avatar attachment & 2.46 & 1.42 \\
Empathy & 3.30 & 1.60 \\
Engagement (GEQ) & 11.43 & 6.01 \\
Positive affect score & 14.51 & 4.56 \\
Negative affect score & 21.07 & 7.39 \\
Guilt score & 9.97 & 4.77 \\
Gaming status & Yes \\
Gamer & 79 & 22 \\
Previous gameplay alignment: Good & 34 & 67 \\
Previous gameplay alignment: Neutral & 34 \\
Previous gameplay alignment: Evil & 57 & 74 \\
\hline
\end{tabular}

Table 3. The three multiple linear regression models with in-game moral alignment.

\begin{tabular}{|c|c|c|c|c|c|c|c|c|}
\hline Regression $n$ models & $\mathbf{R}^{2}$ & $\Delta \mathbf{R}^{2}$ & $p$ & Constant/ significant predictors & B & SE B & $\beta$ & $p$ \\
\hline \multirow[t]{7}{*}{ 1) Real-life morality MFQ } & 0.11 & 0.05 & .090 & Constant & 2.00 & 1.24 & & \\
\hline & & & & $\mathrm{C} / \mathrm{H}$ & 0.82 & 0.31 & 0.32 & .010 \\
\hline & & & & $\mathrm{F} / \mathrm{C}$ & -0.28 & 0.38 & -0.10 & .458 \\
\hline & & & & L/B & 0.03 & 0.35 & 0.01 & .935 \\
\hline & & & & $A / S$ & 0.30 & 0.33 & 0.12 & .373 \\
\hline & & & & $S / D$ & 0.08 & 0.29 & 0.04 & .778 \\
\hline & & & & L/O & -0.20 & 0.27 & -0.08 & .461 \\
\hline \multirow[t]{10}{*}{ 2) Previous gameplay } & 0.37 & 0.30 & $<.001$ & Constant & 6.81 & 1.76 & & \\
\hline & & & & Number of genres played & -0.20 & 0.06 & -0.53 & .001 \\
\hline & & & & Ability & 0.48 & 0.23 & 0.46 & .042 \\
\hline & & & & Gaming status & 0.78 & 0.50 & 0.17 & .124 \\
\hline & & & & Years playing & 0.03 & 0.04 & 0.08 & .538 \\
\hline & & & & Gamer & -0.14 & 0.54 & -0.04 & .797 \\
\hline & & & & Length of time & -0.01 & 0.03 & -0.04 & .771 \\
\hline & & & & Experience & -0.16 & 0.25 & -0.18 & .507 \\
\hline & & & & Previous neutral alignment & -0.66 & 0.39 & -0.15 & .090 \\
\hline & & & & Previous evil alignment & -1.98 & 0.48 & -0.41 & $<.001$ \\
\hline \multirow{8}{*}{$\begin{array}{l}\text { 3) In-game and post-game } \\
\text { measures }\end{array}$} & 0.18 & 0.12 & .008 & Constant & 3.70 & 0.79 & & \\
\hline & & & & Positive affect & 0.04 & 0.09 & 0.09 & .701 \\
\hline & & & & Negative affect & 0.00 & 0.03 & 0.01 & .923 \\
\hline & & & & Guilt scale & 0.02 & 0.09 & 0.04 & .866 \\
\hline & & & & Engagement (GEQ) & -0.08 & 0.04 & -0.24 & .035 \\
\hline & & & & Tangram & 0.08 & 0.04 & 0.18 & .061 \\
\hline & & & & Avatar attachment & -0.26 & 0.15 & -0.20 & .072 \\
\hline & & & & Empathy & 0.36 & 0.13 & 0.31 & .008 \\
\hline
\end{tabular}


Table 4. MFT salience with in-game moral choice and RTs.

\begin{tabular}{|c|c|c|c|c|c|c|c|c|c|}
\hline MFT domain & Salience & $\begin{array}{l}\text { Percentage } \\
\text { of salience }\end{array}$ & $\begin{array}{l}\text { Percentage of in-game } \\
\text { MFT upheld }\end{array}$ & $\chi^{2}(1)$ & $p$ & $V$ & $t(99)$ & $p$ & $d$ \\
\hline $\mathrm{C} / \mathrm{H}$ & Highest & 51.5 & 96.0 & 4.42 & .052 & .21 & -0.59 & .558 & -.12 \\
\hline $\mathrm{F} / \mathrm{C}$ & High & 32.7 & 98.0 & 0.99 & .451 & .10 & 0.81 & .423 & .16 \\
\hline L/O & High & 24.8 & 96.0 & 1.43 & .255 & .12 & 0.08 & .938 & .02 \\
\hline $\mathrm{A} / \mathrm{S}$ & Low & 21.8 & 67.3 & 2.09 & .118 & .14 & 0.54 & .589 & .12 \\
\hline L/B & Low & 29.7 & 69.3 & 0.14 & .440 & .19 & 2.14 & .035 & .43 \\
\hline$S / D$ & Lowest & 58.4 & 91.1 & 3.78 & .051 & .04 & -0.85 & .400 & -.17 \\
\hline
\end{tabular}

Bowman, et al., 2016). Tamborini, Bowman, et al. (2016) suggested differences in MFT domains could be due to how they are processed/accessed. Interestingly, $\mathrm{C} / \mathrm{H}$ and $S / D$ were the quickest domains and at the extreme ends of salience, therefore, this could suggest that the highest and lowest salient domains are processed differently.

The results suggested participants preferred to be helpful both in-game and post-game, which could suggest similarities and/or reciprocal relationship between in-game and post-game behavior (e.g., Ellithorpe et al., 2015). Alternatively, as negative affect was higher than positive affect, this could have led to compensation by being helpful post-game (e.g., Ellithorpe et al., 2015; Gollwitzer \& Melzer, 2012). Furthermore, empathy significantly predicted more pro-social choices and positive in-game alignment. Therefore, empathy could have a specific role in gameplay, which has been found previously (Grizzard, Tamborini, Lewis, Wang, \& Prabhu, 2014; Grizzard, Tamborini, Sherry, \& Weber, 2017). This could suggest the game was emotionally demanding, however, scores on other emotional measures, such as the PANAS, were low. Furthermore, if decisions were being rationalized (as reflected in the time taken), it was unlikely that system 1 was being used, which is more connected to emotional processes, and hence the purposemade game was unlikely to be emotionally demanding (Hartmann, 2011; Kahneman, 2011). In contrast to empathy, game engagement had a significant negative relationship with pro-social choices and in-game alignment. This might suggest a trade-off is made between investment in the game and empathy e.g., empathy vs evil alignment (Triberti et al., 2015). Furthermore, this relates to the choice of the gut or the game, suggested by Joeckel et al. (2012, 2013), if participants chose empathy/gut they would uphold moral choices, whereas if they chose engagement in the game, they would make more violating choices. This trade-off could be explained by the demands of video games, as it demonstrates potential processes and interaction between player and game: i.e., which demand determines how the trade-off is managed/decided and which demands get overridden.

Since the data suggested that overall real-life morality did not predict in-game decision-making, it could indicate that participants were playing to their own preference and/or strategy. This is supported by previous research that found players have a preference for moral decisions, which tend to be pro-social (Lange, 2014; Triberti et al., 2015) and use strategies (Schell, 2014; Sicart, 2010). This could also be represented in the results of previous gameplay, explaining the most variance with evil alignment and ability being significant predictors and suggest previous preferences and strategies. Another possibility is that strategies are related to cognitive demands. Further support could be suggested from the RTs where participants could have been deliberating on these decisions and using system 2 (Hartmann, 2011; Kahneman, 2011; Tamborini, 2011). If system 2 was engaged this could have overridden emotional demands potentially for cognitive demands. Furthermore, these strategies may override/outweigh morality and could relate to moral disengagement and management in gameplay (Hartmann \& Vorderer, 2010; Klimmt, Schmid, Nosper, Hartmann, \& Vorderer, 2006). Another explanation is that the decisions in the purposemade game were being rationalized and perceived as amoral: where participants (consciously or not) did not engage morality. One reason could be that participants were trying to understand new experiences/learn from the game; which could be reflected in L/B reporting the longest RTs as this was likely to be the first decision made.

Cognitive demands, such as skill, have been previously found to overlap with behavioral demands, such as performance in the game (Bowman, Weber, Tamborini, \& Sherry, 2013) and likewise moral agency requires both moral judgment (cognitive) and action (behavior; Bandura, 2002). Eden et al. (2018) highlight the role of social aspects in behavioral affordances. Therefore, the role of empathy and interacting with NPCs in the purposemade game could suggest social demands. Conversely, it is important to note that avatar attachment was low which could have influenced the social interactions and suggested a low social demand (Banks \& Bowman, 2016).

One of the limitations of this study is that it took place in a laboratory and could be different from participants' normal video game experience. This could have also led to social desirability from participants playing the game to meet perceived expectations, rather than how they might normally play games. Additionally, participants could have picked an option based on what they might have found to be entertaining/fun. Also, the majority of participants selected pro-social/upholding choice, therefore, anti-social/violating choices were underrepre- 
sented. Due to time restrictions only NPCs that were required for interactions were included; adding more NPCs can enhance the realism, interactivity, and create a busier VE (Warpefelt \& Verhagen, 2016). Also, participants may have not benefited from making fast decisions, as the game did not specifically facilitate quick responses, which could have influenced the speed of decisions. It is also acknowledged there are limitations within the MFT theory such as modularity of the foundations (Suhler \& Churchland, 2011) and the participant sample only consisting of university students. The results of MFT salience had limitations from how it was calculated, in that the salient domains may not be significantly different from the next highest and lowest domains.

Many of the inconsistencies with previous research could be due to the complex nature of morality containing different components, therefore, highlighting the importance of understanding and mapping the demands of video games, for both commercial and purpose-made games. Tamborini, Bowman, et al. (2016) found that MFT domains influence in-game decisions; however, this influence is affected by the game design. Future research could explore in-game intuitive versus deliberative reasoning, and measure the demands of the purpose-made game to support understanding in-game experiences/decision-making.

\section{Conclusions}

Exploring in-game RTs and moral alignment in a purposemade game with the demands of video games demonstrates the complex nature of in-game moral decisionmaking. The results suggested in-game decisions were mostly pro-social and took between 4-6 seconds, with previous gameplay, in-game, and post-game experiences predicting moral alignment. Overall, real-life morality and salience was not related to in-game decision-making in this study; rather in-game decisions seemed to be more rational and cognitively demanding and thus, not driven by intuitions.

\section{Acknowledgments}

All the participants that took part in the research and those involved with the development of the purposemade game: Davide Melacca, Christos Gatzidis, and Eike Falk Anderson.

\section{Conflict of Interests}

The authors declare no conflict of interests.

\section{References}

American Psychological Association. (2015). Task force on violent media technical report on the review of the violent video game literature. Washington, DC: American Psychological Association. Retrieved from http://www.apa.org/news/press/releases/2015/08/ technical-violent-games.pdf

Bandura, A. (2002). Selective moral disengagement in the exercise of moral agency. Journal of Moral Education, 31(2), 101-119. https://doi.org/10.1080/ 0305724022014322

Banks, J., \& Bowman, N. D. (2016). Emotion, anthropomorphism, realism, control: Validation of a merged metric for player-avatar interaction (PAX). Computers in Human Behavior, 54, 215-223. https://doi.org/ 10.1016/j.chb.2015.07.030

Bowman, N. D. (2016). Video gaming as co-production. In R. Lind (Ed.), Produsing 2.0: The intersection of audiences and production in a digital world (Vol. 2, pp. 107-123). New York, NY: Peter Lang.

Bowman, N. D. (2018). The demanding nature of video game play. In N. D. Bowman (Ed.), Video games: A medium that demands our attention (pp. 1-24). London: Routledge. https//doi.org/10.4324/ 9781351235266

Bowman, N. D., Wasserman, J., \& Banks, J. (2018). Development of the video game demand scale. In $\mathrm{N}$. D. Bowman (Ed.), Video games: A medium that demands our attention (pp. 208-233). London: Routledge. https://doi.org/10.4324/9781351235266

Bowman, N. D., Weber, R., Tamborini, R., \& Sherry, J. (2013). Facilitating game play: How others affect performance at and enjoyment of video games. Media Psychology, 16(1), 39-64. https://doi.org/10.1080/ 15213269.2012.742360

Brockmyer, J. H., Fox, C. M., Curtiss, K. A., McBroom, E., Burkhart, K. M., \& Pidruzny, J. N. (2009). The development of the Game Engagement Questionnaire: A measure of engagement in video game-playing. Journal of Experimental Social Psychology, 45(4), 624-634. https://doi.org/10.1016/j.jesp.2009.02.016

Eden, A., Ewoldsen, D., Lee, J., \& Beyea, D. (2018). Behavioral demands as behavioral affordances in video games. In N. D. Bowman (Ed.), Video games: A medium that demands our attention. (pp. 92-107). London: Routledge. https://doi.org/10.4324/ 9781351235266

Ellithorpe, M. E., Cruz, C., Velez, J. A., Ewoldsen, D. R., \& Bogert, A. K. (2015). Moral license in video games: When being right can mean doing wrong. $C y$ berpsychology, Behavior, and Social Networking, 18(4), 203-207. https://doi.org/10.1089/cyber.2014.0599

Elson, M., \& Quandt, T. (2016). Digital games in laboratory experiments: Controlling a complex stimulus through modding. Psychology of Popular Media Culture, 5(1), 52-65. https://doi.org/10.1037/ ppm0000033

Gollwitzer, M., \& Melzer, A. (2012). Macbeth and the joystick: Evidence for moral cleansing after playing a violent video game. Journal of Experimental Social Psychology, 48(6), 1356-1360. https://doi.org/10.1016/ j.jesp.2012.07.001

Graham, J., Haidt, J., \& Nosek, B. (2008). Questionnaires. 
MoralFoundations.org. Retrieved from http://www. moralfoundations.org/questionnaires

Graham, J., Nosek, B. A., Haidt, J., lyer, R., Koleva, S., \& Ditto, P. H. (2011). Mapping the moral domain. Journal of Personality and Social Psychology, 101(2), 366-385. https://doi.org/10.1037/a0021847

Grizzard, M., Tamborini, R., Lewis, R. J., Wang, L., \& Prabhu, S. (2014). Being bad in a video game can make us more morally sensitive. CyberPsychology, Behavior \& Social Networking, 17(8), 499-504. https://doi.org/10.1089/cyber.2013.0658

Grizzard, M., Tamborini, R., Sherry, J. L., \& Weber, R. (2017). Repeated play reduces video games' ability to elicit guilt: Evidence from a longitudinal experiment. Media Psychology, 20(2), 267-290. https://doi.org/ 10.1080/15213269.2016.1142382

Haidt, J. (2012). The righteous mind: Why good people are divided by politics and religion. New York, NY: Vintage.

Haidt, J., \& Joseph, C. (2008). The moral mind: How five sets of innate intuitions guide the development of many culture-specific virtues, and perhaps even modules. In P. Carruthers, S. Laurence, \& S. Stich (Eds.), The innate mind, volume 3: Foundations and the future (pp. 367-391). Oxford: Oxford University Press. https://doi.org/10.1093/acprof:oso/ 9780195332834.003.0019

Hartmann, T. (2011). Players' experiential and rational processing of virtual violence. In K. Poels \& S. Malliet (Eds.), Moral issues in digital game play (pp. 135-150). Leuven: Acco.

Hartmann, T., Krakowiak, K. M., \& Tsay-Vogel, M. (2014). How violent video games communicate violence: $A$ literature review and content analysis of moral disengagement factors. Communication Monographs, 81(3), 310-332. https://doi.org/10.1080/03637751. 2014.922206

Hartmann, T., Toz, E., \& Brandon, M. (2010). Just a game? Unjustified virtual violence produces guilt in empathetic players. Media Psychology, 13(4), 339-363. https://doi.org/10.1080/15213269.2010.524912

Hartmann, T., \& Vorderer, P. (2010). It's okay to shoot a character: Moral disengagement in violent video games. Journal of Communication, 60(1), 94-119. https://doi.org/10.1111/j.1460-2466.2009.01459.x

Haviv, S., \& Leman, P. J. (2002). Moral decisionmaking in real life: Factors affecting moral orientation and behaviour justification. Journal of moral education, 31(2), 121-140. https://doi.org/10.1080/ 03057240220143241

Hodge, S. E. (2018). Press trigger for morality: An exploration into the role of moral development, moral decision-making and video game play (Unpublished Doctoral thesis). Bournemouth University, Poole, UK. Hodge, S. E., McAlaney, J., Gatzidis, C., Anderson, E. F., Melacca, D., \& Taylor, J. (2018). Applying psychological theory to in-game moral behaviors through the development of a purpose-made game. In N. D. Bowman (Ed.), Video games: A medium that demands our attention. (pp. 108-125). London: Routledge. https://doi.org/10.4324/9781351235266

Huskey, R., Craighead, B., Miller, M. B., \& Weber, R. (2018). Does intrinsic reward motivate cognitive control? A naturalistic-fMRI study based on the synchronization theory of flow. Cognitive, Affective, \& Behavioral Neuroscience, 18(5), 902-924. https://doi.org/ 10.3758/s13415-018-0612-6

Joeckel, S., Bowman, N. D., \& Dogruel, L. (2012). Gut or game? The influence of moral intuitions on decisions in video games. Media Psychology, 15(4), 460-485. https://doi.org/10.1080/15213269.2012.727218

Joeckel, S., Bowman, N. D., \& Dogruel, L. (2013). The influence of adolescents' moral salience on actions and entertainment experience in interactive media. Journal of Children and Media, 7(4), 480-506. https:// doi.org/10.1080/17482798.2013.781513

Kahneman, D. (2011). Thinking, fast and slow. New York, NY: Farrar, Straus and Giroux.

Klimmt, C., Schmid, H., Nosper, A., Hartmann, T., \& Vorderer, P. (2006). How players manage moral concerns to make video game violence enjoyable. Communications, 31(3), 309-328. https://doi.org/10. 1515/COMMUN.2006.020

Lange, A. (2014). 'You're just gonna be nice': How players engage with moral choice systems. Journal of Games Criticism, 1(1), 1-16. Retrieved from http:// gamescriticism.org/articles/lange-1-1

Lewis, M., \& Jacobson, J. (2002). Game engines in scientific research: Introduction to special issue. Communications of the ACM, 45(1), 27-31. https://doi.org/ $10.1145 / 502269.502288$

Lombard, M., Ditton, T. B., \& Weinstein, L. (2007). Measuring presence: The temple presence inventory (TPI). Matthew Lombard. Retrieved from http:// matthewlombard.com/research/p2_ab.html

Mohseni, M. R., Liebold, B., \& Pietschmann, D. (2015). Extensive modding for experimental game research. In P. Lankoski \& S. Björk (Eds.), Game research methods: An overview (pp. 323-340). Pittsburgh, PA: ETC Press.

Narvaez, D., \& Rest, J. (1995). The four components of acting morally. In W. Kurtines \& J. Gewirtz (Eds.), Moral behavior and moral development: An introduction (pp. 385-400). New York, NY: McGraw-Hill.

Palmer, J. (2015). Time for a timer! Effective use of timers in game design. Gamasutra. Retrieved from https://www.gamasutra.com/blogs/JackPalmer/ 20151019/256526/Time_for_a_timer_Effective_ use_of_timers_in_game_design.php

Rutledge, P. B. (2013). Arguing for media psychology as a distinct field. In K. E. Dill (Ed.), The Oxford handbook of media psychology, (pp. 43-61). New York, NY: Oxford University Press.

Saleem, M., Anderson, C. A., \& Barlett, C. P. (2015). Assessing helping and hurting behaviors through the Tangram help/hurt task. Personality and Social Psychology Bulletin, 41(10), 1345-1362. https://doi.org/ $10.1177 / 0146167215594348$ 
Schell, J. (2014). The art of game design: A book of lenses. Boca Raton, FL: CRC Press.

Sicart, M. (2010). Wicked games: on the design of ethical game play. In B. T. Christensen, S. Boztepe, \& T. Kristensen (Eds.), Proceedings of the 1st DESIRE network conference on creativity and innovation in design (pp. 101-111). Aarhus: DESIRE Network.

Stromer-Galley, J. (2004). Interactivity-as-product and interactivity-as-process. The Information Society, 20(5), 391-394. https://doi.org/10.1080/ 01972240490508081

Suhler, C. L., \& Churchland, P. (2011). Can innate, modular "foundations" explain morality? Challenges for Haidt's moral foundations theory. Journal of cognitive neuroscience, 23(9), 2103-2116. https://doi.org/ 10.1162/jocn.2011.21637

Swink, S. (2009). Game feel: A game designer's guide to virtual sensation. Burlington: Morgan Kaufmann Publishers.

Taber, K. S. (2018). The use of Cronbach's alpha when developing and reporting research instruments in science education. Research in Science Education, 48(6), 1273-1296. https://doi.org/10.1007/s11165016-9602-2

Tamborini, R. (2011). Moral intuition and media entertainment. Journal of Media Psychology: Theories, Methods and Applications, 23, 39-45. https://doi. org/10.1027/1864-1105/a000031

Tamborini, R. (2012). A model of intuitive morality and exemplars. In R. Tamborini (Ed.), Media and the moral mind (pp. 43-74). London: Routledge. https://doi. org/10.4324/9780203127070

Tamborini, R., \& Bowman, N. D. (2010). Presence in video games. In C. C. Bracken \& P. Skalski, (Eds.), Immersed in media: Telepresence in everyday life (pp. 87-109). New York, NY: Routledge.

Tamborini, R., Bowman, N. D., Prabhu, S., Hahn, L., Klebig, B., Grall, C., \& Novotny, E. (2016). The effect of moral intuitions on decisions in video game play: The impact of chronic and temporary intuition accessibility. New Media \& Society, 1-17. https://doi.org/10. 1177/1461444816664356

Tamborini, R., Lewis, R. J., Prabhu, S., Grizzard, M., Hahn, L., \& Wang, L. (2016). Media's influence on the accessibility of altruistic and egoistic motivations. Communication Research Reports, 33(3), 177-187. https:// doi.org/10.1080/08824096.2016.1186627

Triberti, S., Villani, D., \& Riva, G. (2015). Moral positioning in video games and its relation with dispositional traits: The emergence of a social dimension. Computers in Human Behavior, 50, 1-8. https://doi.org/ 10.1016/j.chb.2015.03.069

Warpefelt, H., \& Verhagen, H. (2016). A typology of nonplayer characters. Proceedings of 1st international joint conference of DiGRA and FDG. Dundee: Digital Games Research Association and Society for the Advancement of the Science of Digital Games.

Watson, D., \& Clark, L. A. (1999). The PANAS-X: Manual for the positive and negative affect scheduleexpanded form. Iowa City, IA: University of lowa. https://doi.org/10.17077/48vt-m4t2

Weaver, A. J., \& Lewis, N. (2012). Mirrored morality: An exploration of moral choice in video games. $C y$ berPsychology, Behavior \& Social Networking, 15(11), 610-614. https://doi.org/10.1089/cyber.2012.0235

Whelan, R. (2008). Effective analysis of reaction time data. The Psychological Record, 58(3), 475-482. https://doi.org/10.1007/BF03395630

\section{About the Authors}
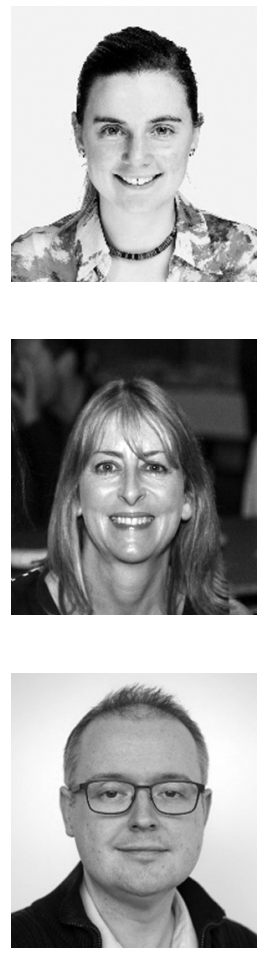

Sarah E. Hodge (PhD) has a background in psychology, and she completed her PhD at Bournemouth University exploring the role of moral development and decision making in video games. Since her $\mathrm{PhD}$, Sarah has continued to explore morality in video games, including the application of the Moral Foundations Theory in gaming research. Sarah has also developed her research to explore the overlap between gaming and gambling, the role of gender and gaming, and how to psychologically support users.

Jacqui Taylor (Associate Professor) is a Chartered Psychologist and Associate Fellow of the BPS. Jacqui completed her PhD at the University of Portsmouth and this explored the social psychological impacts of the internet on communication and group processes. Her current research has developed from this interest and now includes topics such as online deviance, digital addiction, behavior change and cybersecurity, and ways to enhance adolescents and student wellbeing online.

John McAlaney (Associate Professor) is a Chartered Psychologist and Chartered Scientist. He completed his PhD at the University of West of Scotland, where he explored the social psychological factors associated with risky alcohol use. He has since expanded his research to include psychological determinants of other risk behaviors including gambling, digital addiction, and cybersecurity, with a focus on how research in this area can be applied to improve psychological wellbeing. 


\section{Appendix}

\section{Appendix A: The Purpose-Made Game and the Six MFT In-Game Scenarios}

\subsection{Game Synopsis}

The purpose-made game was designed to reduce and remove biases, as such the game does not have a strong focus on narrative. The environment and NPCs were designed and made to look as realistic as possible. Due to the large size of the purpose-made game files there is a dedicated computer lab to run these types of experiments. At the beginning of the game participants were given instructions on how to play the game, including that they will be making decisions in the game. There was also a short tutorial at the beginning to demonstrate the only options that participants will be presented within the game. The keyboard controlled the movements of the participants in-game avatar's body (only avatar's hands seen by participants) and to interact with NPCs. The mouse controlled where the avatar's head was looking. Left and right buttons on the mouse were how participants made the in-game choice and corresponded to the in-game presentation of the choices.

Each scenario was in the form of short vignettes. The text in italics was presented to participants in the game; the in-game vignette followed by the two options for each MFT domain. The descriptions that were used for validating the vignette have also been included to add context, since this would have been seen in the gameplay.

\subsubsection{Scenario Care/Harm}

Description: You see a person is injured and holding their abdomen, crying while they are slumped on the ground.

In-Game vignette: [Sobbing] Ouch, ouch I am injured; these heavy books fell on me, ouch.

Pro-social choice-Upholds MFT: Help the injured person, pick up books and help them up.

Anti-social choice-Violates MFT: Hurt the injured person more, drop more books on them.

\subsubsection{Scenario Fairness/Cheating}

Description: You see a person is struggling to share out coins equally (with another), because some are damaged, smaller, and worthless.

In-Game vignette: [Sighs] These coins are difficult to share out equally, some are damaged and worthless.

Pro-social choice-Upholds MFT: Help restore the coins' value and divide them equally.

Anti-social choice-Violates MFT: Damage more coins and divide the coins unequally.

\subsubsection{Scenario Loyalty/Betrayal}

Description: You see a person is giving others each a chest containing a written private promise, and all swear to secrecy.

In-Game vignette: [Whispers] In these chests is a private promise, all agreed to swear to secrecy.

Pro-social choice-Upholds MFT: Put locks on the chests and protect the promise.

Anti-social choice-Violates MFT: Destroy the chests, open and display the promise.

\subsubsection{Scenario Authority/Subversion}

Description: You see a General is holding a pair of boots which are scuffed and orders them to be sorted out.

In-Game vignette: [Commands] You! This scuffed pair of boots on the windowsill, sort them out!

Pro-social choice-Upholds MFT: Mend and polish the boots, to sort them out.

Anti-social choice-Violates MFT: Damage the boots more, not sorting them out.

\subsubsection{Scenario Sanctity/Degradation}

Description: You see a person is trying to maintain the waste system that contains sewage, which is leaking out.

In-Game vignette: [Sighs] This waste system needs to be maintained and is leaking sewage. 
Pro-social choice-Upholds MFT: Fix and strengthen the waste system and cleanse the area.

Anti-social choice-Violates MFT: Destroy and damage the waste system causing more leakage.

\subsubsection{Scenario Liberty/Oppression}

Description: You see a person is trying to construct and open a stage to allow for people's free expression.

In-Game vignette: [Sighs] This stage is difficult to construct and open for people's free expression.

Pro-social choice-Upholds MFT: Help create the stage to allow for free expression.

Anti-social choice-Violates MFT: Destroy the stage to control and stop free expression.

\section{Appendix B: Predictor Variables}

Table 5. Individual predictor variables for the three regression models.

\begin{tabular}{|c|c|c|c|}
\hline Regression model & Predictor variables & Response/range & $\mathbf{N}$ \\
\hline \multirow[t]{7}{*}{ Real-life morality } & MFQ32 scores: & & \\
\hline & - Care/Harm (C/H) & $0-5$ & 101 \\
\hline & - Fairness/Cheating (F/C) & $0-5$ & 101 \\
\hline & - Authority/Subversion (A/S) & $0-5$ & 101 \\
\hline & - Sanctity/Degradation (S/D) & $0-5$ & 101 \\
\hline & - (In-group) Loyalty/ Betrayal (L/B) & $0-5$ & 101 \\
\hline & - Liberty/Oppression (L/O) scores & $0-5$ & 101 \\
\hline \multirow{10}{*}{$\begin{array}{l}\text { Previous video } \\
\text { game play }\end{array}$} & Plays video games & Yes/No & 101 \\
\hline & Would describe themselves as a gamer & Yes/No & 101 \\
\hline & Previous good alignment & Yes/No & 101 \\
\hline & Previous bad alignment* & Yes/No & 101 \\
\hline & Previous neutral alignment & Yes/No & 101 \\
\hline & Length of time & $0-52.50$ hours & 101 \\
\hline & Years playing & $0-26$ years & 100 \\
\hline & Number of genres played* & $0-19$ & 101 \\
\hline & Experience & $0-7$ & 101 \\
\hline & Ability* & $0-7$ & 101 \\
\hline \multirow[t]{11}{*}{ Post-game questions } & Empathy* (2 item): & $0-7$ & 101 \\
\hline & 1. How much did you empathize with the characters in the game? & & \\
\hline & 2. How connected did you feel with the other characters in the game? & & \\
\hline & Avatar Attachment (3 item): & $0-7$ & 101 \\
\hline & 1. How much did you identify with your avatar? & & \\
\hline & 2. How attached did you feel with your avatar? & & \\
\hline & 3. Did you feel that avatar was you? & & \\
\hline & Tangrams help/hurt task & $-9-9$ & 101 \\
\hline & Engagement (GEQ)* & $0-32$ & 101 \\
\hline & PANAS-X & $10-50$ & 101 \\
\hline & — Guilt score & $6-30$ & 101 \\
\hline
\end{tabular}

Notes: Forced entry method was used on all three regressions as no hierarchy was applied to the input of variables. ${ }^{*}$ denotes a significant predictor.

\section{Appendix C: Liberty/Oppression Scale}

The Liberty/Oppression scale items followed the same format as the rest of the MFQ (Graham, Haidt, \& Nosek, 2008)

\subsection{Part 1-Moral Relevance}

Whether or not someone was controlled by another person.

Whether or not someone was restricted by their government. 
Whether or not someone was free to choose how to live their life.

\subsection{Part 2-Moral Judgment}

People should not be oppressed by their government.

People should not be forbidden to make their own decisions.

People have the right to disagree with those in power.

Although the Cronbach's Alpha was satisfactory/acceptable $(\alpha=.62)$ and similar to the other MFQ domains (Graham et al., 2008; Taber, 2018). It is acknowledged this is a new scale and has not had the level of testing and validation of the other MFT domains. For more information on the development of the scale see Hodge (2018).

\section{References}

Graham, J., Haidt, J., \& Nosek, B. (2008). Questionnaires. MoralFoundations.org. Retrieved from http://www. moralfoundations.org/questionnaires

Hodge, S. E. (2018). Press trigger for morality: An exploration into the role of moral development, moral decision-making and video game play (Unpublished doctorate dissertation). Bournemouth University, Poole, UK.

Taber, K. S. (2018). The use of Cronbach's alpha when developing and reporting research instruments in science education. Research in Science Education, 48(6), 1273-1296. https://doi.org/10.1007/s11165-016-9602-2 